\title{
Differential On-On Keying: A Robust Non-Coherent Digital Modulation Scheme
}

\author{
Georges Kaddoum*, Mohammed F. A. Ahmed*† and Tareq Y. Al-Naffourił§ \\ *University of Québec, École de Technologie Supérieure, Montréal, Canada \\ $\dagger$ Electrical Engineering Department, Assiut University, Assiut, Egypt \\ $\ddagger$ King Abdullah University of Science and Technology (KAUST), Thuwal, KSA \\ §Electrical Engineering Department, King Fahd University of Petroleum and Minerals, Dhahran, KSA \\ Email: georges.kaddoum@etsmtl.ca, m.ahmed@ieee.org, tareq.alnaffouri@kaust.edu.sa
}

\begin{abstract}
A robust digital modulation scheme, called differential on-on keying (DOOK), is presented in this paper which outperforms the conventional on-off keying (OOK). In this scheme, a sinusoidal signal is transmitted during the first half of the bit duration while a replica or an inverted version of the sinusoidal signal is transmitted during the second half for logic one or logic zero, respectively. Non-coherent receiver correlates the two halves of the received signal over half bit duration to construct a decision variable. Bit error performance is analyzed over AWGN and Rayleigh fading channels and compared to the conventional OOK.
\end{abstract}

Index Terms-DOOK, WSNs, Robust non-coherent modulation, Performance analysis.

\section{INTRODUCTION}

In recent years, wireless communications are being increasingly used for power-constrained applications like wireless sensor networks (WSNs). As a result, energy-efficiency became as important as spectral efficiency and bit error rate (BER) in designing wireless transmission schemes. Specifically, modulation techniques with low energy consumption are considered for such scenarios even at the expense of suboptimal spectral efficiency and BER performance. For instance, on-off keying (OOK) modulation has gained some attention lately in WSNs [1]. While other modulation techniques can achieve better BER performance than OOK [2], the energy efficient and low-complexity non-coherent detectors of the OOK make it attractive for low power scenarios. In the OOK scheme, a sinusoidal signal is transmitted to represent logic one and no signal is transmitted to represent logic zero. For equiprobable bit stream, the transmitter consumes only half of the energy as compared to phase shift keying (PSK) or frequency shift keying (FSK). A coherent receiver is not commonly used for OOK because it requires channel estimation and carrier synchronization which add complexity to the system. On the other hand, non-coherent receivers employing envelop detector [1], [2] or energy detector [3] have low complexity and thus are used for OOK. While it avoids synchronization requirements, the non-coherent detection adopted in OOK has a different challenge, namely, finding the optimal threshold for decision making that minimizes the BER. An approximate average BER expression is developed in [4] for multi-user scenario with direct sequence code division multi access (DS-CDMA) and a numerical algorithm is proposed to compute the optimal threshold. The decision threshold in this case depends on the average channel coefficient of the desired signal and both the average and variance of the sum of all interfering signals. In [5], different iterative numerical algorithms are proposed to compute the decision threshold for the same scenario under Rayleigh fading conditions. Alternatively, different schemes are proposed to avoid threshold estimation like block-coded OOK [3] and soft decision based OOK detection [6].In this paper, we propose differential onon keying (DOOK) as a low power modulation scheme with energy detection that uses fixed zero threshold. This simplifies the system implementation since no threshold optimization is needed. In this scheme, a sinusoidal signal is transmitted during the first half of the bit duration for both logic zero and logic one. During the second half of the bit duration, a replica of the sinusoidal signal is transmitted for logic one, while an inverted version of the signal is transmitted for logic zero. The receiver correlates both the first and second halves of the received signal over half bit duration to generate a decision variable and retrieve the transmitted bit. This non-coherent DOOK receiver avoids the need for channel gain or noise power estimators. A BER expression is derived for the DOOK over additive white Gaussian noise (AWGN) and Rayleigh fading channel which is then compared to the conventional OOK with envelop and energy detectors.

\section{System Model}

In this section, we briefly present the OOK scheme then introduce the DOOK scheme.

1) OOK scheme: The carrier in the OOK transmitter is modulated by the transmitted bits. For logic one, the modulator is enabled and a sinusoidal signal with energy $E_{b}$ is transmitted during the bit duration $T_{b}$, otherwise, the modulator is disabled for logic zero and no signal is transmitted. For the $i$ th bit, the $k$ th sample of the transmitted OOK signal, $k=1, \ldots, N$, is given by

$$
u_{k, i}=b_{i} \sqrt{\frac{2 E_{b}}{T_{b}}} \sin \left(2 \pi f_{c} \frac{k}{N} T_{b}\right),
$$

where $b_{i}=\{0,1\}$ is the information bit, $f_{c}$ is the carrier frequency, and $N$ is the number of samples per bit. The bit duration is found as $T_{b}=N / f_{s}$ where $f_{s}$ is the sampling 
frequency. Assume a fading channel, the $k$ th sample of the received signal is given by

$$
v_{k, i}=h_{i} u_{k, i}+n_{k},
$$

where $n_{k}$ is complex AWGN with zero mean and variance $N_{0}$ and $h_{i}$ is the channel coefficient during the $i$ th bit. The channel coefficient can follow any wireless channel fading model like Rayleigh or Rician and it is assumed to be constant during bit duration $T_{b}$. At the receiver, either envelope or energy detection can be used. An envelope detector samples the absolute value (i.e. the envelope) of the received signal once per bit [2] and the envelope sample is then used as a decision variable $D_{\mathrm{env}, i}$. For an ideal channel, i.e. no AWGN or fading, the decision variable takes on the values $\left\{0, \sqrt{2 E_{b} / T_{b}}\right\}$ for $b_{i}=\{0,1\}$, respectively. However, it is actually a random variable in the presence of the AWGN and channel fading which can be expressed as [2]

$$
D_{\mathrm{env}, i}=\sqrt{\left|h_{i} b_{i} \sqrt{2 E_{b} / T_{b}}+n_{R}\right|^{2}+\left|n_{I}\right|^{2}} .
$$

where $n_{R}$ and $n_{I}$ are the real and imaginary parts of $n_{k}$. The decision variable is compared to a predefined threshold $\beta_{\text {env }}$ and the $i$ th bit is detected as

$$
\hat{b}_{i}= \begin{cases}0, & D_{\mathrm{env}, i} \leq \beta_{\mathrm{env}} \\ 1, & D_{\mathrm{env}, i}>\beta_{\mathrm{env}}\end{cases}
$$

where $\beta_{\mathrm{env}}=\sqrt{N_{0}\left(2+E_{b} /\left(2 T_{b} N_{0}\right)\right)}$ [2]. Alternatively, when an energy detector is used, the received signal passes through a squaring device and the output is integrated over the bit duration $T_{b}$ to obtain the decision variable which is expressed as

$$
\begin{aligned}
D_{\text {eng }, i} & =\mathbb{R}\left\{\sum_{k=1}^{N}\left(h_{i} b_{i} \sqrt{\frac{2 E_{b}}{T_{b}}} \sin \left(2 \pi f_{c} \frac{k}{N} T_{b}\right)+n_{k}\right)^{2}\right\} \\
& =\left|h_{i}\right|^{2} b_{i}^{2} E_{b}+\sum_{k=1}^{N} n_{k}^{2} \\
& +\sum_{k=1}^{N} 2 n_{k} h_{i} b_{i} \sqrt{\frac{2 E_{b}}{T_{b}}} \sin \left(2 \pi f_{c} \frac{k}{N} T_{b}\right)
\end{aligned}
$$

where $\mathbb{R}\{\cdot\}$ is the real part of complex number. The values of the decision variable in an ideal channel would be $\left\{0, E_{b}\right\}$ for $b_{i}=\{0,1\}$, respectively. However, in the presence of AWGN and fading channel, the threshold is function of the noise variance and the channel fading. In this paper, the threshold is approximated as $\beta_{\text {eng }}=N_{0}+0.5\left|h_{i}\right|^{2} E_{b}$ [7].

2) DOOK scheme: The DOOK system model is shown in Fig. 1. In this scheme, each bit $b_{i}=\{-1,+1\}$ is represented by two sinusoidal signals during the bit duration $T_{b}$. The first sinusoidal signal occupies the first half of the bit duration, i.e. the $k$ th samples for $0 \leq k \leq N / 2$, and represents the reference signal, while the second sinusoidal signal occupies the second half, i.e. the $k$ th samples for $N / 2<k \leq N$, and carries the information bit (hereafter it will be referred to as the databearing signal). If $b_{i}=1$, the data-bearing signal is the same

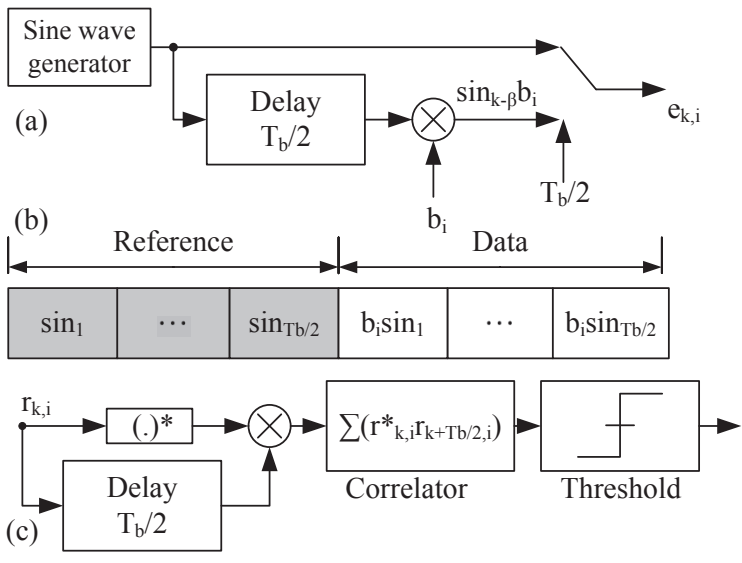

Fig. 1. (a) DOOK modulator, (b) DOOK frame, (c) DOOK receiver

as the reference signal, and if $b_{i}=-1$, the data-bearing signal is an inverted version of the reference signal. Thus, during the $i$ th bit, the $k$ th sample of the transmitted signal is given by

$$
x_{k, i}=\left\{\begin{array}{lll}
\sqrt{\frac{E_{b}}{T_{b}}} & \sin \left(2 \pi f_{c} \frac{k}{N} T_{b}\right), & \text { for } 1<k \leq N / 2, \\
\sqrt{\frac{E_{b}}{T_{b}}} b_{i} & \sin \left(2 \pi f_{c} \frac{k}{N} T_{b}\right), & \text { for } N / 2<k \leq N .
\end{array}\right.
$$

For fair comparison, the carrier energy over $T_{b}$ is set to $E_{b} / 2$ to make the DOOK transmitter consume the same energy as the OOK scheme. At the receiver, the $k$ th sample of the received signal is given by

$$
r_{k, i}=h_{i} x_{k, i}+n_{k}
$$

As shown in Fig. 1, the received signal is delayed by half bit duration $T_{b} / 2$ and both the received and delayed versions of the signal are used to construct the decision variable $D_{i}$. Specifically, for the $i$ th bit, the first half of the received signal $r_{k, i}, 1 \leq k \leq N / 2$, is correlated with the complex conjugate of the second half of the received signal $r_{k, i}^{*}, N / 2<k \leq N$ and the result is summed over half bit duration $T_{b} / 2$. Hence, the decision variable for the $i$ th bit is given by

$$
\begin{aligned}
D_{i} & =\mathbb{R}\left\{\sum_{k=1}^{\frac{N}{2}}\left(h_{i} \sqrt{\frac{E_{b}}{T_{b}}} \sin \left(2 \pi f_{c} \frac{k}{N} T_{b}\right)+n_{k}\right)\right. \\
& \left.\times\left(h_{i} \sqrt{\frac{E_{b}}{T_{b}}} b_{i} \sin \left(2 \pi f_{c} \frac{k+\frac{N}{2}}{N} T_{b}\right)+n_{k+\frac{N}{2}}\right)^{*}\right\}
\end{aligned}
$$

It is clear from (8) that the decision variable $D_{i}$ takes the values $\left\{-E_{b} / 4, E_{b} / 4\right\}$ for $b_{i}=\{-1,1\}$, respectively, for the ideal channel. Therefore, the received bits are estimated by comparing the decision variable $D_{i}$ to zero, which is a fixed threshold, as

$$
\hat{b}_{i}=\operatorname{sign}\left(D_{i}\right)
$$

where $\operatorname{sign}(\cdot)$ is the sign function. 


\section{DOOK SYSTEM ANALYSIS}

In this section, we first show the benefit of using differential scheme for data transmission and explain the advantage of employing DOOK rather than OOK. Next, we derive the BER performance of the DOOK over AWGN and fading channels.

1) $\mathrm{DOOK}$ versus $\mathrm{OOK}$ : In the conventional OOK, energy detection is equivalent to correlating the received signal with itself over the bit duration $T_{b}$. Assume the simple case of AWGN channel. For logic zero, only the noise is available at the receiver input. The detector correlates this noise with itself producing a non-negative decision variable, corresponds to the noise energy, which increases as the signal-to-noise ratio (SNR) decreases. For logic one, the decision variable contains both the carrier and noise energies. Therefore, the optimum threshold is function of the SNR and, accordingly, requires to be optimized for each SNR to obtain the best performance. On the contrary, DOOK scheme uses a fixed zero threshold because the decision variable is constructed in a differential fashion by correlating the two halves of the received signal over $T_{b} / 2$ period. Expanding (8), we have

$$
\begin{aligned}
D_{i} & =\mathbb{R}\left\{\sum _ { k = 1 } ^ { \frac { N } { 2 } } \left(\left|h_{i}\right|^{2} \frac{E_{b}}{T_{b}} b_{i} \sin ^{2}\left(2 \pi f_{c} \frac{k}{N} T_{b}\right)+n_{k} n_{k+\frac{N}{2}}^{*}\right.\right. \\
& +h_{i} \sqrt{\frac{E_{b}}{T_{b}}} \sin \left(2 \pi f_{c} \frac{k}{N} T_{b}\right) n_{k+\frac{N}{2}}^{*} \\
& \left.\left.+h_{i}^{*} \sqrt{\frac{E_{b}}{T_{b}}} b_{i} \sin \left(2 \pi f_{c} \frac{k+\frac{N}{2}}{N} T_{b}\right) n_{k}\right)\right\} \\
& =\left|h_{i}\right|^{2} b_{i} \frac{E_{b}}{4}+\mathbb{R}\left\{\sum_{k=1}^{\frac{N}{2}} n_{k} n_{k+\frac{N}{2}}^{*}\right\} \\
& +\sum_{k=1}^{\frac{N}{2}}\left(h_{i} \sqrt{\frac{E_{b}}{T_{b}}} \sin \left(2 \pi f_{c} \frac{k}{N} T_{b}\right) n_{k+\frac{N}{2}}^{*}\right. \\
& \left.+h_{i}^{*} \sqrt{\frac{E_{b}}{T_{b}}} b_{i} \sin \left(2 \pi f_{c} \frac{k+\frac{N}{2}}{N} T_{b}\right) n_{k}\right)
\end{aligned}
$$

Note that the last three terms in the latter equation have zero mean because of the independent zero mean noise. Also, the last two terms in (10) corresponds to the last term in (5). The multiplied independent noise samples, i.e. $\mathbb{R}\left\{\sum_{k=1}^{\frac{N}{2}} n_{k} n_{k+\frac{N}{2}}^{*}\right\}$, can be positive, negative, or zero with a smaller or equal value to the corresponding term in the OOK case given in (5), i.e.

$$
\mathbb{R}\left\{\sum_{k=1}^{\frac{N}{2}} n_{k} n_{k+\frac{N}{2}}^{*}\right\} \leq \mathbb{R}\left\{\sum_{k=1}^{N} n_{k}^{2}\right\},
$$

which results in lower noise contribution in the received DOOK signal. Additionally, the decision variable in the DOOK is symmetric around zero for any SNR value and channel fading and, consequently, no adaptive threshold is required. However, the Euclidean distance between the two values of the decision variable in the ideal channel for the
DOOK scheme, $E_{b} / 2$, is smaller than the OOK one, $E_{b}$. We show in the simulation section that the lower noise energy contribution in the DOOK decision variable according to (11) compensates this Euclidean distance reduction.

While the performance of the OOK improves with increasing the SNR in the AWGN channel because the noise component in (5) reduces, the situation is completely different for fading channels. At the receiver, the squaring operation results in a squared channel coefficient as shown in (5). Therefore, squared Rayleigh fading, for example, has an exponential distribution with most of its values are concentrated near zero. Hence, even without AWGN, the values of the decision variable corresponding to logic one are scaled to values towards zero. Since the threshold is a finite positive value in the OOK scheme, the channel scaling degrades the error performance for any SNR which leads to BER flooring. The same situation occurs for the Rician or any other fading distribution. On the other hand, the decision variable for the DOOK is symmetrically distributed around zero and the channel scaling affects both the negative and positive values in the same way. Thus, while channel fading degrades the BER performance for the DOOK, it does not result in error flooring.

2) BER performance analysis: According to the central limit theorem, the decision variable $D_{i}$, given a transmitted bit $b_{i}$, is Gaussian distributed. Gaussian approximation of the decision variable is commonly used in the analysis of the OOK with energy detection [8], [9]. Therefore, the total BER can be expressed as

$$
\begin{aligned}
& \mathrm{BER}=\frac{1}{2} \operatorname{Pr}\left(D_{i}<0 \mid b_{i}=+1\right)+\frac{1}{2} \operatorname{Pr}\left(D_{i}>0 \mid b_{i}=-1\right) \\
& =\frac{1}{2} \operatorname{erfc}\left(\frac{E\left\{D_{i} \mid b_{i}=+1\right\}}{\sqrt{2 \operatorname{Var}\left\{D_{i} \mid b_{i}=+1\right\}}}\right)
\end{aligned}
$$

where $\operatorname{erfc}(x)$ is the complementary error function defined as $\operatorname{erfc}(x) \equiv \frac{2}{\sqrt{\pi}} \int_{x}^{\infty} e^{-t^{2}} d t$. The later expression requires computing the mean and variance of the decision variable $D_{i}$. For a given bit $b_{i}$, the mean $E\left\{D_{i}\right\}$ of the decision variable can be found as

$$
E\left\{D_{i}\right\}=b_{i}\left|h_{i}\right|^{2} \frac{E_{b}}{4} .
$$

Also, the variance $\operatorname{Var}\left\{D_{i}\right\}$ of the decision variable can be written as

$$
\begin{aligned}
& \operatorname{Var}\left\{D_{i}\right\}=E\left\{D_{i}^{2}\right\}-E\left\{D_{i}\right\}^{2} \\
= & E\left\{\left(\mathbb{R}\left\{\sum_{k=1}^{\frac{N}{2}} n_{k} n_{k+\frac{N}{2}}^{*}\right\}\right)^{2}\right\} \\
+ & E\left\{\left(\mathbb{R}\left\{\sum_{k=1}^{\frac{N}{2}} h_{i} \sqrt{\frac{E_{b}}{T_{b}}} \sin \left(2 \pi f_{c} \frac{k}{N} T_{b}\right) n_{k+\frac{N}{2}}^{*}\right\}\right)^{2}\right\} \\
+ & E\left\{\left(\mathbb{R}\left\{\sum_{k=1}^{\frac{N}{2}} h_{i}^{*} \sqrt{\frac{E_{b}}{T_{b}}} b_{i} \sin \left(2 \pi f_{c} \frac{k+\frac{N}{2}}{N} T_{b}\right) n_{k}\right\}\right)^{2}\right\} \\
= & T_{b} \frac{N_{0}^{2}}{8}+\left|h_{i}\right|^{2} E_{b} \frac{N_{0}}{4},
\end{aligned}
$$




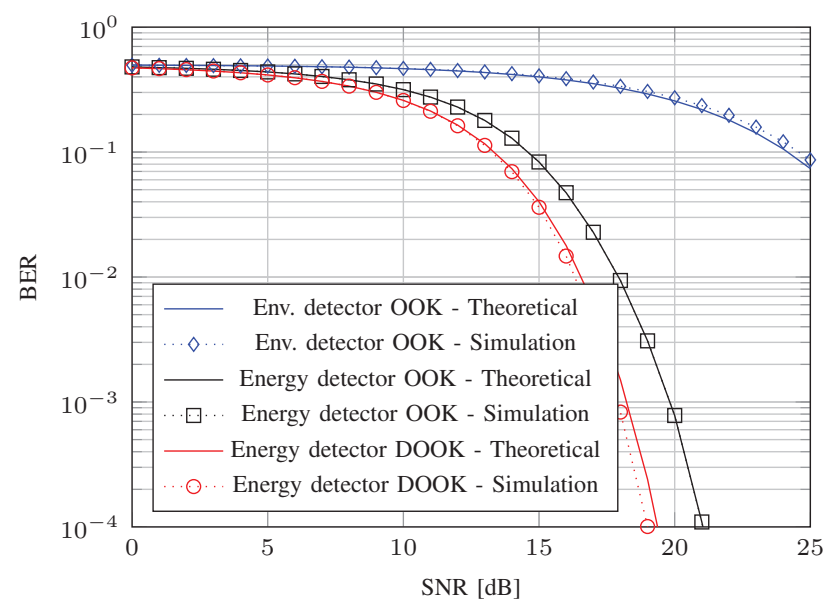

Fig. 2. BER for DOOK and OOK over AWGN: $N=100$.

where the last equality is obtained after performing some straightforward manipulations. Substituting (12) and (13) in (12), the BER for a given channel coefficient $h_{i}$ can be expressed as

$$
\begin{aligned}
\mathrm{BER} & =\frac{1}{2} \operatorname{erfc}\left(\frac{\left|h_{i}\right|^{2} E_{b}}{2 \sqrt{T_{b} N_{0}^{2}+2\left|h_{i}\right|^{2} E_{b} N_{0}}}\right) \\
& =\frac{1}{2} \operatorname{erfc}\left(\left(\frac{4 T_{b}}{\gamma_{i}^{2}}+\frac{8}{\gamma_{i}}\right)^{-\frac{1}{2}}\right)
\end{aligned}
$$

where $\gamma_{i}=\frac{\left|h_{i}\right|^{2} E_{b}}{N_{0}}$ is the instantaneous received SNR. Finally, the average BER is

$$
\overline{\mathrm{BER}}=\frac{1}{2} \int_{0}^{\infty} \operatorname{erfc}\left(\left(\frac{4 T_{b}}{\gamma_{i}^{2}}+\frac{8}{\gamma_{i}}\right)^{-\frac{1}{2}}\right) d \gamma_{i},
$$

which can be evaluated by numerical integration.

\section{Simulation RESUlts}

Fig. 2 shows the DOOK system performance under AWGN channel (i.e substituting $h_{i}=1$ in (14)) for $N=100$ and the corresponding performance of the conventional OOK for both envelope detection [2] and energy detection [7]. The theoretical expressions are plotted with solid lines and the simulations are plotted with dashed lines and marks. The results show that the DOOK outperforms the OOK with both detection methods. This is mainly due to the reduced noise effects as shown in (11). Fig. 3 shows the simulations and theoretical BER expressions given in (15) under Rayleigh channel and compare it to the OOK scheme. As expected, the proposed DOOK scheme outperforms the conventional noncoherent OOK without any need of channel gain or noise power estimation. Another important advantage shown in this figure is the robustness of the DOOK system against channel fading. We can clearly see that the BER of the OOK has an error floor for high SNR. This happens because the threshold is positive and the channel gain scales the decision variable to values smaller than the predefined threshold.

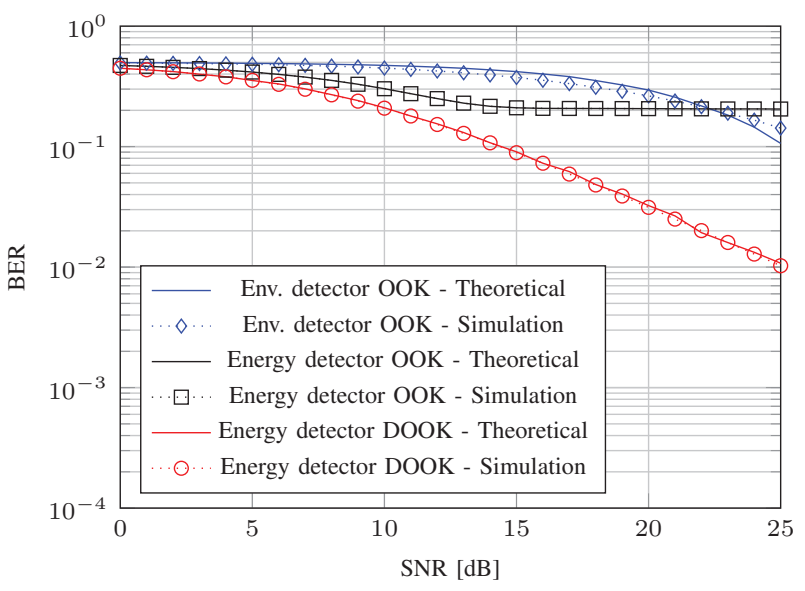

Fig. 3. BER for DOOK and OOK over Rayleigh channels: $N=100$.

\section{CONCLUSion}

A new robust non-coherent DOOK scheme was presented in this paper. The analysis showed that this scheme outperforms the conventional OOK over AWGN and fading channels with the same transmitted bit energy and without the need for channel gain or noise power estimation. The DOOK scheme is robust against channel fading since it has a decision variable which is symmetrically distributed around zero and uses fixed zero threshold.

\section{ACKNOWLEDGEMENTS}

This work is supported by the Natural Sciences and Engineering Research Council (NSERC), Canada, discovery grant no. 435243-2013, and by King Abdulaziz City of Science and Technology (KACST), KSA, grant no. AT-34-345.

\section{REFERENCES}

[1] Q. Tang, S. K. S. Gupta, and L. Schwiebert, "BER performance analysis of an on-off keying based minimum energy coding for energy constrained wireless sensor applications," in IEEE International Conference on Communications 2005, ICC 2005, vol. 4, May 2005, pp. 2734-2738.

[2] M. Schwartz, W. R. Bennett, and S. Stein, Communication Systems and Techniques. IEEE Press, 1996.

[3] X. Cheng, Y. Guan, and Y. Gong, "Thresholdless energy detection for ultra-wideband block-coded OOK signals," Electronics Letters, vol. 44, no. 12, pp. 755-756, Jun. 2008.

[4] M. D'Angelo and C. Fischione, "How to select the OOK detection threshold in wireless Ad Hoc and sensor networks," in IEEE 69th Vehicular Technology Conference 2009, VTC Spring 2009, Apr. 2009.

[5] D. Katselis, C. Fischione, and H. Hjalmarsson, "Efficient OOK/DSCDMA detection threshold selection," in American Control Conference (ACC), 2013, Jun. 2013, pp. 3523-3528.

[6] P. Zhang, F. M. J. Willems, and L. Huang, "Investigations of non-coherent OOK based schemes with soft and hard decisions for WSNs," in 49th Annual Allerton Conference on Communication, Control, and Computing (Allerton) 2011, Sep. 2011, pp. 1702-1709.

[7] P. Humblet and M. Azizoglu, "On the bit error rate of lightwave systems with optical amplifiers," Journal of Lightwave Technology, vol. 9, no. 11, pp. 1576-1582, Nov. 1991

[8] M. Sahin, I. Guvenc, and H. Arslan, "Optimization of energy detector receivers for UWB systems," in IEEE 61st Vehicular Technology Conference 2005, VTC 2005-Spring, vol. 2, May 2005, pp. 1386-1390.

[9] A. Anttonen, A. Mammela, and A. Kotelba, "Sensitivity of energy detected multilevel PAM systems to threshold mismatch," in IEEE International Conference on Ultra-Wideband 2008, ICUWB 2008, vol. 1, Sep. 2008, pp. 165-168. 